\title{
The high-density limit of two-electron systems: Results from the extended Overhauser approach
}

\author{
Paola Gori-Giorgi and Andreas Savin \\ Laboratoire de Chimie Théorique, \\ CNRS UMR7616, Université Pierre et Marie Curie, \\ 4 Place Jussieu, F-75252 Paris, France
}

September 28, 2018

\begin{abstract}
The "extended Overhauser model" [Overhauser, A.W. Can. J. Phys. 1995, 73, 683] for the calculation of the spherically and system-averaged pair density (APD) has been recently combined with the Kohn-Sham equations to yield realistic APD and correlation energies. In this work we test this approach in the high-density (weakly-correlated) limit of the He isoelectronic series and of the Hooke's atom isoelectronic series. Unlike many of the commonly used energy functionals, the Overhauser approach yields accurate correlation energies for both series.
\end{abstract}

\section{Introduction}

Kohn-Sham (KS) Density Functional Theory ${ }^{1-3}$ (DFT) is nowadays one of the most popular methods for electronic structure calculations both in chemistry and solid-state physics, thanks to its combination of low computational cost and reasonable performances. The accuracy of a KS-DFT result is limited by the approximate nature of the exchange-correlation energy density functional $E_{x c}[n]$. Simple approximations (local-density approximation and generalized gradient corrections) for $E_{x c}[n]$ provide practical estimates of thermodynamical, structural and spectroscopic properties of atoms, molecules and solids. However, with the current approximations, KS-DFT is still lacking in several aspects, in particular it fails to handle near-degeneracy correlation effects (rearrangement of electrons within partially filled shells) and to recover long-range van der Waals interaction energies. The inaccuracy of KS-DFT stems from our lack of knowledge of $E_{x c}[n]$, and much effort is put nowadays in finding new approximations to this term (for recent reviews, see, e.g., Refs. 2-4). A popular trend in the development of new KS $E_{x c}[n]$ is the use of the exact exchange functional $E_{x}[n]$ (in terms of the KS orbitals), and thus the search for an approximate, compatible, correlation functional $E_{c}[n]$. 
An exact expression for $E_{c}[n]$ is the coupling-constant integral ${ }^{5,6}$

$$
E_{c}[n]=\int_{0}^{\lambda_{\text {phys }}} d \lambda \int_{0}^{\infty} d r_{12} 4 \pi r_{12}^{2} f_{c}^{\lambda}\left(r_{12}\right) \frac{\partial w^{\lambda}\left(r_{12}\right)}{\partial \lambda},
$$

where the interaction between the electrons is adiabatically turned on from $w^{\lambda=0}\left(r_{12}\right)=0$ to the Coulomb repulsion $w^{\lambda=\lambda_{\text {phys }}}\left(r_{12}\right)=1 / r_{12}$ by varying a real parameter $\lambda$ (typical examples are $w^{\lambda}\left(r_{12}\right)=\lambda / r_{12}$, with $\lambda_{\text {phys }}=1$, or $w^{\lambda}\left(r_{12}\right)=\operatorname{erf}\left(\lambda r_{12}\right) / r_{12}$, with $\left.\lambda_{\text {phys }}=\infty\right)$. The one-electron density $n(\mathbf{r})$ is (ideally) kept independent of $\lambda$ and equal to the one of the physical system by means of a suitable external potential $v^{\lambda}(\mathbf{r})$. In Eq. (1) the correlation part of the spherically and system-averaged pair density (intracule density) $f_{c}^{\lambda}\left(r_{12}\right)$ is defined as follows. For each $\lambda$, take the square of the many-electron wavefunction $\Psi^{\lambda}$ ground-state of the hamiltonian $H^{\lambda}$,

$$
H^{\lambda}=-\sum_{i=1}^{N} \frac{\nabla_{i}^{2}}{2}+\frac{1}{2} \sum_{i \neq j=1}^{N} w^{\lambda}\left(\left|\mathbf{r}_{i}-\mathbf{r}_{j}\right|\right)+\sum_{i=1}^{N} v^{\lambda}\left(\mathbf{r}_{i}\right),
$$

and integrate it over all variables but the scalar electron-electron distance $r_{12}=$ $\left|\mathbf{r}_{1}-\mathbf{r}_{2}\right|$,

$$
f^{\lambda}\left(r_{12}\right)=\frac{N(N-1)}{2} \sum_{\sigma_{1} \ldots \sigma_{N}} \int\left|\Psi^{\lambda}\left(\mathbf{r}_{12}, \mathbf{R}, \mathbf{r}_{3}, \ldots, \mathbf{r}_{N}\right)\right|^{2} \frac{d \Omega_{\mathbf{r}_{12}}}{4 \pi} d \mathbf{R} d \mathbf{r}_{3} \ldots d \mathbf{r}_{N}
$$

where $\mathbf{R}=\left(\mathbf{r}_{1}+\mathbf{r}_{2}\right) / 2$. The correlation part $f_{c}^{\lambda}\left(r_{12}\right)$ is then defined as $f_{c}^{\lambda}\left(r_{12}\right)=$ $f^{\lambda}\left(r_{12}\right)-f_{\mathrm{KS}}\left(r_{12}\right)$, where the intracule density of the KS system is $f_{\mathrm{KS}}\left(r_{12}\right)=$ $f^{\lambda=0}\left(r_{12}\right)$ (and yields the Hartree plus the exchange energy).

The traditional DFT approach to the construction of approximate $E_{c}[n]$ is based on the idea of universality. For example, the familiar local-density approximation (LDA) consists in transfering, in each point of space, the pair density from the uniform electron gas to obtain an approximation for $f_{c}^{\lambda}\left(r_{12}\right)$ in Eq. (10). In a couple of recent papers, ${ }^{7-9}$ we have started to explore a different way of constructing $E_{c}[n]$, based on an "average pair density functional theory" (APDFT), which was inspired by the seminal work of Overhauser ${ }^{10}$ and its subsequent extensions. ${ }^{11-13}$ In this approach, we solve a set of radial (onedimensional) Schrödinger-like equations that give, in principle, the exact $f^{\lambda}\left(r_{12}\right)$ along the DFT adiabatic connection. In practice, this formalism contains an unknown effective electron-electron interaction that needs to be approximated. The APDFT equations must be solved for each system, and combined selfconsistently with the KS equations. ${ }^{9}$ Preliminary applications of this approach, combined with a simple approximation ${ }^{7}$ for the effective electron-electron interaction that enters in the formalism, gave accurate intracule densities $f\left(r_{12}\right)$ and correlation energies $E_{c}[n]$ for the He isoelectronic series. ${ }^{7,9}$

Katriel et al. ${ }^{14}$ have recently tested most of the currently available correlation energy functionals in the high-density (weakly-correlated) limit of the $\mathrm{He}$ and of the Hooke's atom isoelectronic series, finding that, while several functionals are accurate for the He sequence, none is satisfactory for the Hooke's atom 
series. Motivated by their findings, in this work we compute the correlation energy and the intracule density in the high-density limit of the two series via the APDFT approach,${ }^{7-9}$ finding accurate results in both cases.

The paper is organized as follows. In the next Sec. 2 we recall the basic equations that define the high-density limit of the He and Hooke's atom sequences, to which we apply, in Sec. 3 and 4, the formalism of Refs. 7-9 to compute the intracule density and the correlation energy. In Sec. 5 we also analyze the failure of LDA in this limit from the point of view of $f\left(r_{12}\right)$. The last Sec. 6 is devoted to conclusions.

\section{The high-density limit of the He and Hooke's atom isoelectronic series}

The two hamiltonians analyzed in this paper read

$$
\begin{aligned}
H & =-\frac{\nabla_{1}^{2}}{2}-\frac{\nabla_{2}^{2}}{2}+v\left(r_{1}\right)+v\left(r_{2}\right)+\frac{1}{r_{12}}, \\
v(r) & = \begin{cases}-\frac{Z}{r} & \text { He series } \\
\frac{1}{2} k r^{2} & \text { Hooke's atom series. }\end{cases}
\end{aligned}
$$

We are interested in the high-density (weakly-correlated) limit, which corresponds to $Z \rightarrow \infty$ and $k \rightarrow \infty$. By switching to scaled coordinates $\mathbf{s}=\mathbf{r} / \alpha$, with $\alpha=Z^{-1}$ (He series) and $\alpha=k^{-1 / 4}$ (Hooke's series), both hamiltonians have the form

$$
H=\frac{1}{\alpha^{2}}\left(-\frac{\nabla_{\mathbf{s}_{1}}^{2}}{2}-\frac{\nabla_{\mathbf{s}_{2}}^{2}}{2}+\tilde{v}\left(s_{1}\right)+\tilde{v}\left(s_{2}\right)+\frac{\alpha}{s_{12}}\right) \equiv \frac{1}{\alpha^{2}}\left(\tilde{H}_{0}+\alpha \tilde{H}_{1}\right),
$$

where $\tilde{v}(s)=-1 / s$ for the He series, and $\tilde{v}(s)=s^{2} / 2$ for the Hooke's atom series. We thus study pertubatively the system described by $\tilde{H}_{0}+\alpha \tilde{H}_{1}$.

The order zero of the one-electron density $n(r)$ and of the intracule density $f\left(r_{12}\right)$, in scaled units, is simply

$$
\begin{aligned}
n^{(0)}(s) & = \begin{cases}\frac{2}{\pi} e^{-2 s} & (\text { He }) \\
\frac{2}{\pi^{3 / 2}} e^{-s^{2}} & (\text { Hooke })\end{cases} \\
f^{(0)}\left(s_{12}\right) & = \begin{cases}\frac{1}{24 \pi}\left(3+6 s_{12}+4 s_{12}^{2}\right) e^{-2 s_{12}} & \text { (He) } \\
\frac{1}{(2 \pi)^{3 / 2}} e^{-s_{12}^{2} / 2} & \text { (Hooke) }\end{cases}
\end{aligned}
$$

These functions are correctly normalized, so that if we switch back to coordinates $\mathbf{r}$ we have $n^{(0)}(r)=\alpha^{-3} n^{(0)}\left(s=\alpha^{-1} r\right)$, etc.

The first-order correction to the scaled density, $n(s)=n^{(0)}(s)+\alpha n^{(1)}(s)+\ldots$ is given by

$$
n^{(1)}(s)=2 n^{(0)}(s) \chi(s),
$$

where $^{15}$

$$
\chi(s)=-\frac{23}{32}-\frac{e^{-2 s}}{4}-\frac{3}{8} \gamma+\frac{3}{16} \frac{1-e^{-2 s}}{s}+\frac{5}{8} s+\frac{3}{8} \operatorname{Ei}(-2 s)-\frac{3}{8} \ln (s),
$$


for the He isoelectronic series, with $\gamma=0.577216 .$. ,

$$
\operatorname{Ei}(-x)=-\int_{x}^{\infty} \frac{e^{-t}}{t} d t
$$

$\operatorname{and}^{16}$

$$
\begin{aligned}
\chi(s) & =\frac{\operatorname{erf}(s)}{s}-\frac{\sqrt{2}(1+\ln 2)}{\sqrt{\pi}}-\frac{1}{s \sqrt{\pi}} \int_{0}^{\infty} d x\left(e^{-(x-s)^{2}}-e^{-(x+s)^{2}}\right) \\
& \times\left[e^{x^{2} / 2} \operatorname{erfc}\left(\frac{x}{\sqrt{2}}\right)+\sqrt{2} x \int_{0}^{x / \sqrt{2}} d t e^{t^{2}} \operatorname{erfc}(t)\right],
\end{aligned}
$$

for the Hooke's atom isoelectronic series.

By definition, the Kohn-Sham hamiltonian describes a non-interacting system that has the same density of the physical, interacting, system. Thus, the first-order change in the electron density of Eq. (8) corresponds to a first-order change in the KS system. Therefore, we write the scaled intracule $f\left(s_{12}\right)$ up to orders $\alpha$ as

$$
f\left(s_{12}\right)=f^{(0)}\left(s_{12}\right)+\alpha\left[f_{\mathrm{KS}}^{(1)}\left(s_{12}\right)+f_{c}^{(1)}\left(s_{12}\right)\right]+O\left(\alpha^{2}\right),
$$

where we have separated the first-order correction into a Kohn-Sham part and a correlation part. The KS part $f_{\mathrm{KS}}^{(1)}$ is entirely determined by the first-order density $n^{(1)}$ of Eq. (요),

$$
f_{\mathrm{KS}}^{(1)}\left(s_{12}\right)=\int d \mathbf{s} n^{(0)}\left(\mathbf{s}+\mathbf{s}_{12}\right) n^{(0)}(s) \chi(s),
$$

and is reported in Appendix $\mathrm{A}$ in analytic form for the He isoelectronic series, while is obtained numerically for the case of the Hooke's series.

The total first-order intracule $f^{(1)}=f_{\mathrm{KS}}^{(1)}+f_{c}^{(1)}$ is known analytically in the case of the Hooke's series, ${ }^{16}$

$f^{(1)}\left(s_{12}\right)=\frac{2 e^{-s_{12}^{2} / 4}}{(2 \pi)^{3 / 4}}\left[1-\frac{1+\ln 2}{\sqrt{2 \pi}}+\frac{1}{s_{12}}-\frac{e^{s_{12}^{2} / 2}}{s_{12}} \operatorname{erfc}\left(\frac{s_{12}}{\sqrt{2}}\right)+\sqrt{2} \int_{0}^{s_{12} / \sqrt{2}} e^{t^{2}} \operatorname{erfc}(t) d t\right]$.

\section{Effective equations for $f\left(r_{12}\right)$ in the high-density limit}

\subsection{Formalism}

We are interested in calculating $f_{c}^{(1)}$ and the corresponding second-order correlation energy $E_{c}^{(2)}$ with the method of Refs. 7-9, in which the intracule density $f\left(r_{12}\right)$ of the physical system is obtained from a set of effective equations, which for two-electron systems reduce to

$$
\left[-\nabla_{r_{12}}^{2}+w_{\text {eff }}\left(r_{12}\right)\right] \psi\left(r_{12}\right)=\epsilon \psi\left(r_{12}\right),
$$


with $f\left(r_{12}\right)=\left|\psi\left(r_{12}\right)\right|^{2}$. Equation (15) can be derived by considering 8,9 a set of Hamiltonians characterized by a real parameter $\xi$,

$$
H^{\xi}=-\sum_{i=1}^{N} \frac{\nabla_{i}^{2}}{2}+\frac{1}{2} \sum_{i \neq j=1}^{N} w^{\xi}\left(\left|\mathbf{r}_{i}-\mathbf{r}_{j}\right|\right)+\xi \sum_{i=1}^{N} v_{n e}\left(\mathbf{r}_{i}\right), \quad f^{\xi}\left(r_{12}\right)=f\left(r_{12}\right) \forall \xi
$$

that describe a set of systems in which the external potential is turned off as $\xi \rightarrow 0$, and the intracule density is kept fixed, equal to the one of the physical system, by means of a suitable electron-electron interaction $w^{\xi}\left(r_{12}\right)$. In the case $N=2$, when $\xi=0$ we have a translationally-invariant system (the center-ofmass degree of freedom is described by a plane wave) of two fermions in a relative bound state (similar to the case of positronium, but with a different interaction). This relative bound state is such that the square of the wavefunction for the relative coordinate $r_{12}$ is equal to $f\left(r_{12}\right)$ of the starting physical system, and is thus described by Eq. (15).$^{8,9}$ For more than two electrons, in the case of a confined system (atom, molecule), the limit $\xi \rightarrow 0$ in Eq. (16) describes a cluster of fermions, and Eq. (15) becomes an approximation ${ }^{8,9,17}$ for the internal degrees of freedom of the cluster.

Here we focus on the high-density limit of the hamiltonians of Eq. (5) and we thus stick to the case $N=2$. In general, the effective electron-electron interaction $w_{\text {eff }}\left(r_{12}\right)$ in Eq. (15) is not known, and must be approximated. In the case of the He series, we have found ${ }^{7-9}$ that a simple approximation based on the original idea of Overhauser ${ }^{10,11}$ gives very accurate results for $2 \leq Z \leq 10$. In what follows we analyze the performance of the same approximation in the very $Z \rightarrow \infty$ limit, and we extend our study to the $k \rightarrow \infty$ limit of the Hooke's atom series. Of course, in the special case of the Hooke's series, the hamiltonian (5) is exactly separable into center-of-mass and relative coordinates, so that the exact $w_{\text {eff }}\left(r_{12}\right)$ is directly available. However, the point here is to check whether the same approximate $w_{\text {eff }}\left(r_{12}\right)$ that accurately describes the He series is capable to yield also good results for the Hooke's series, since this seems to be not the case for the currently available correlation energy functionals. ${ }^{14}$

The construction of an approximation for the e-e effective potential $w_{\text {eff }}$ starts with the decomposition ${ }^{7-9}$

$$
w_{\text {eff }}\left(r_{12}\right)=w_{\text {eff }}^{\mathrm{KS}}\left(r_{12}\right)+w_{\text {eff }}^{c}\left(r_{12}\right),
$$

where $w_{\mathrm{eff}}^{\mathrm{KS}}=\nabla^{2} \sqrt{f_{\mathrm{KS}}} / \sqrt{f_{\mathrm{KS}}}$ is the potential that generates the Kohn-Sham $f_{\mathrm{KS}}$ via Eq. (15), and $w_{\text {eff }}^{c}\left(r_{12}\right)$ is a correlation potential that needs to be approximated. In the usual DFT language, Eq. (17) implies that we are treating exchange exaclty.

In scaled units $\mathbf{s}$, using standard perturbation theory we obtain the equation for the first-order contribution to $f$ [see Eq. [12)], that separates into the KohnSham and the correlation parts:

$$
\begin{gathered}
{\left[-\nabla^{2}+w_{\mathrm{eff}}^{\mathrm{KS}(0)}-\epsilon^{(0)}\right] \psi_{\mathrm{KS}}^{(1)}=\left[\epsilon_{\mathrm{KS}}^{(1)}-w_{\mathrm{eff}}^{\mathrm{KS}(1)}\right] \psi^{(0)}} \\
{\left[-\nabla^{2}+w_{\mathrm{eff}}^{\mathrm{KS}(0)}-\epsilon^{(0)}\right] \psi_{c}^{(1)}=\left[\epsilon_{c}^{(1)}-w_{\mathrm{eff}}^{c(1)}\right] \psi^{(0)},}
\end{gathered}
$$


where $\psi^{(0)}=\sqrt{f^{(0)}}, f_{\mathrm{KS}}^{(1)}=2 \psi^{(0)} \psi_{\mathrm{KS}}^{(1)}, f_{c}^{(1)}=2 \psi^{(0)} \psi_{c}^{(1)}$, and

$$
\begin{array}{lll}
w_{\mathrm{eff}}^{\mathrm{KS}(0)}\left(s_{12}\right)= & \frac{2\left(8 s_{12}^{4}-8 s_{12}^{3}-38 s_{12}^{2}-36 s_{12}-9\right)}{\left(4 s_{12}^{2}+6 s_{12}+3\right)^{2}}-1 \text { (He series) }(20) \\
w_{\mathrm{eff}}^{\mathrm{KS}(0)}\left(s_{12}\right)=\frac{s_{12}^{2}}{4} & \text { (Hooke's series). (21) }
\end{array}
$$

In Eq. (18), $f_{\mathrm{KS}}^{(1)}$ is exatly known for both series, so that we can also obtain $w_{\mathrm{eff}}^{\mathrm{KS}(1)}$ by inversion.

We thus concentrate on the correlation part, since we want to test approximations for $w_{\text {eff }}^{c}$. Defining $u_{c}(x)=x \psi_{c}^{(1)}(x)$ and $u_{0}(x)=x \psi^{(0)}(x)$, we have

$$
\left[\frac{d^{2}}{d x^{2}}-w_{\mathrm{eff}}^{\mathrm{KS}(0)}+\epsilon^{(0)}\right] u_{c}=\left[w_{\mathrm{eff}}^{c(1)}-\epsilon_{c}^{(1)}\right] u_{0} .
$$

Following the method of Refs. 15, 18, 19 we look for a solution of the kind $u_{c}(x)=u_{0}(x) y(x)$. The function $y(x)$ is then given by

$$
y(x)=\int_{0}^{x} \frac{d x^{\prime}}{u_{0}^{2}\left(x^{\prime}\right)} \int_{0}^{x^{\prime}} u_{0}^{2}\left(x^{\prime \prime}\right)\left[w_{\mathrm{eff}}^{c(1)}\left(x^{\prime \prime}\right)-\epsilon_{c}^{(1)}\right] d x^{\prime \prime}+C_{2} .
$$

The constant $C_{2}$ is fixed by requiring the proper normalization,

$$
\int_{0}^{\infty} f_{c}^{(1)}(x) x^{2} d x=0 \Rightarrow \int_{0}^{\infty} y(x) u_{0}^{2}(x) d x=0 .
$$

The other integration constant has been fixed in Eq. (23) by setting equal to zero an unphysical term $C_{1} \int^{x} u_{0}^{-2}\left(x^{\prime}\right) d x^{\prime}$ that would make $u_{c}(x)$ diverge for large $x$.

\subsection{Testing approximations: the Overhauser potential}

In Refs. 7-9 an approximation for $w_{\text {eff }}^{c}$ was built as an average "Overhausertype" potential, ${ }^{10,11}$

$$
w_{\mathrm{eff}}^{c}\left(r_{12}\right) \approx\left(\frac{1}{r_{12}}+\frac{r_{12}^{2}}{2 \bar{r}_{s}^{3}}-\frac{3}{2 \bar{r}_{s}}\right) \theta\left(\bar{r}_{s}-r_{12}\right)
$$

where $\theta(x)$ is the Heaviside step function and $\bar{r}_{s}$ is related to the average density, or, better to the dimension of the system. For two-electron atoms it was simply estimated as $^{7}$

$$
\bar{r}_{s}=\left(\frac{4 \pi}{3} \bar{n}\right)^{-1 / 3},
$$

where

$$
\bar{n}=\frac{1}{N} \int d \mathbf{r} n(\mathbf{r})^{2} .
$$

The idea beyond this approximation is the following. The e-e correlation potential $w_{\text {eff }}^{c}\left(r_{12}\right)$ changes the Kohn-Sham $f$ into the physical one, and must 


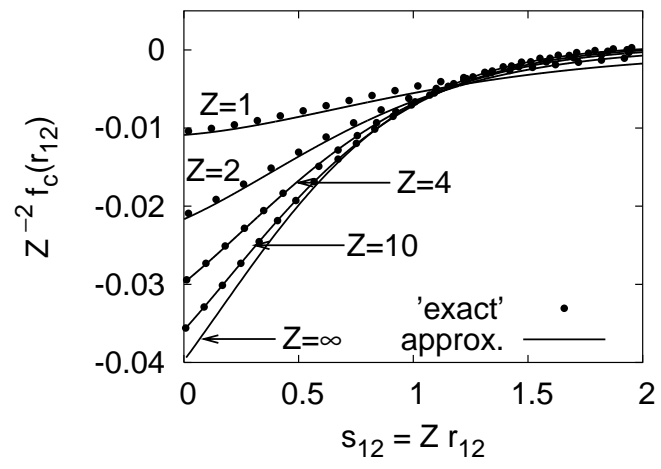

Figure 1: The correlation part of the intracule density, $f_{c}=f-f_{\mathrm{KS}}$, divided by $Z^{2}$, as a function of the scaled variable $s_{12}=Z r_{12}$ for the He isoelectronic series. The "exact" results are obtained from the accurate wavefunctions of Ref. 20. Approximate results at finite $Z$ using the "Overhauser model" are taken from Ref. 7. The $Z=\infty$ result corresponds to Eq. (23) with the potential of Eq. (28).

thus keep the information on the one-electron density (which is the same in the two systems) while turning on the e-e interaction $1 / r_{12}$. In Eqs. (25)-(27) this information is approximately kept via the average density $\bar{n}$.

In scaled units, the Overhauser potential to first order in $\alpha$, to be used in Eq. (19), becomes

$$
w_{\mathrm{eff}}^{c(1)}\left(s_{12}\right) \approx\left(\frac{1}{s_{12}}+\frac{s_{12}^{2}}{2 \bar{s}_{s}^{3}}-\frac{3}{2 \bar{s}_{s}}\right) \theta\left(\bar{s}_{s}-s_{12}\right),
$$

where, if we adopt the prescription of Eqs. (26)-(27), $\bar{s}_{s}=3^{1 / 3}+O(\alpha)$ for the He series and $\bar{s}_{s}=(3 \sqrt{\pi})^{1 / 3}+O(\alpha)$ for the Hooke's atom series.

Equation (23) with the potential of Eq. (28) can be evaluated analytically as a function of $s_{12}$ and $\bar{s}_{s}$ for both series, although the final expressions are cumbersome and will not be reported here. The resulting $f_{c}^{(1)}$ for the He series is shown in Fig. 1, together with the corresponding scaled quantity, $Z^{-2} f_{c}(s / Z)$, for some finite $Z$. [Since $f_{c}^{(1)}(s)=\lim _{Z \rightarrow \infty} Z f_{c}(s)$, and $f_{c}(s)=Z^{-3} f_{c}(s / Z)$, the quantity to be compared with $f_{c}^{(1)}(s)$ is $Z^{-2} f_{c}(s / Z)$.] For finite $Z$ we show both the "exact" result ${ }^{20}$ and the approximate result ${ }^{7}$ obtained with the Overhausertype potential of Eqs. (25)-(27). We see that the $Z$ dependence of the shortrange part of $f_{c}$ is very well captured by this simple approximation. Figure 1 also suggests that the $Z \rightarrow \infty$ limit of the short-range part of $f_{c}$ is well described by this approach. In Fig. 2 we show the result for $f_{c}^{(1)}$ in the case of the Hooke's series from the Overhauser potential compared to the exact one, finding very accurate agreement.

The KS and the correlation components of $f^{(1)}$ are shown in Fig. 3 for both series. We see that in the case of the He series the KS and the correlation 


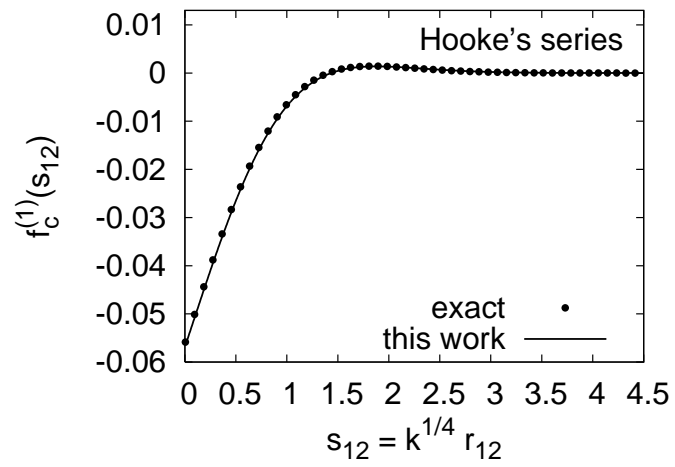

Figure 2: The correlation part of the first-order intracule, $f_{c}\left(s_{12}\right)$ [see Eq. (12)], for the Hooke's series. The exact values are compared with the results from the Overhauser-type approximation of Eq. (28).
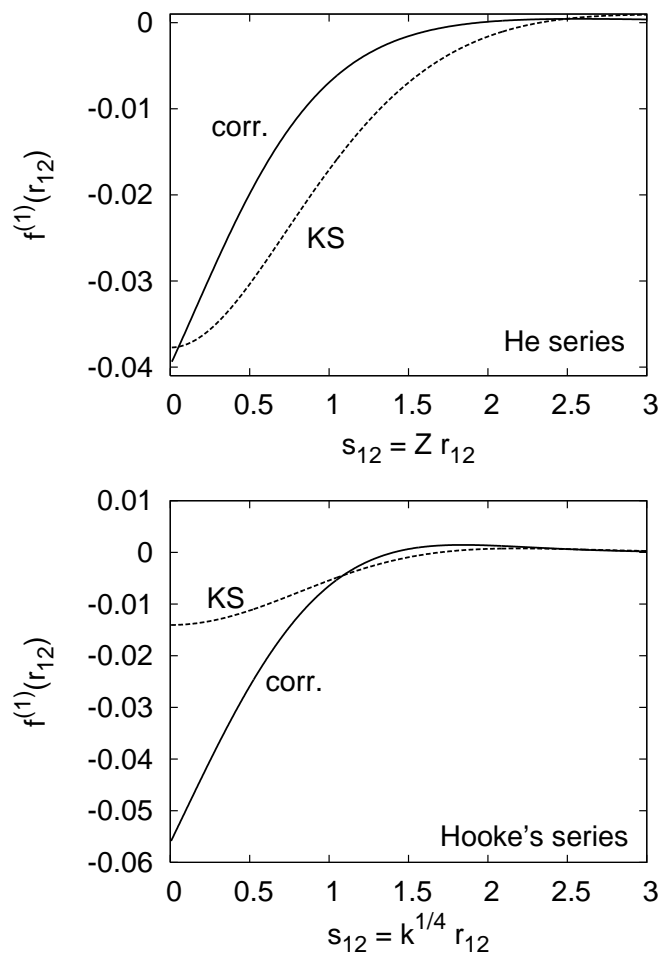

Figure 3: The decomposition of the first-order intracule intracule density $f^{(1)}\left(s_{12}\right)$ [see Eq. (12)]: the Kohn-Sham part and the correlation part. 
parts have roughly the same depth, while in the case of the Hooke's series the correlation part is much deeper than the KS one. This is due to the fact that the $\mathrm{KS}$ part gives the change in the e-e distance probability distribution only due to the first-order change in the one-electron density. In the case of the Hooke's series the first-order change in the density is much smaller, since the harmonic confining external potential is stronger than the Coulombic one. Indeed, the function $\chi(s)$ of Eq. (8) in the case of the He series is about twice the one for the Hooke's atom series.

\section{Adiabatic connection and correlation energy}

The APD $f_{c}^{(1)}\left(s_{12}\right)$ gives the correlation contribution to second order to the expectation $\left\langle V_{e e}\right\rangle$ of the Coulomb electron-electron repulsion operator, $V_{e e}=$ $1 / r_{12}$

$$
\left\langle V_{e e}\right\rangle=\frac{1}{\alpha^{2}}\left[\alpha\left\langle V_{e e}\right\rangle^{(1)}+\alpha^{2}\left\langle V_{e e}\right\rangle^{(2)}+O\left(\alpha^{3}\right)\right],
$$

where $\left\langle V_{e e}\right\rangle^{(2)}=\left\langle V_{e e}\right\rangle_{\mathrm{KS}}^{(2)}+\left\langle V_{e e}\right\rangle_{c}^{(2)}$, and

$$
\left\langle V_{e e}\right\rangle_{c}^{(2)}=\int_{0}^{\infty} 4 \pi s_{12} f_{c}^{(1)}\left(s_{12}\right) d s_{12}
$$

Our $f_{c}^{(1)}$ from the Overhauser potential give $\left\langle V_{e e}\right\rangle_{c}^{(2)}=-0.10256 \mathrm{Ha}$ for the He sequence (to be compared with the exact ${ }^{21}$ value, $-0.09333 \mathrm{Ha}$ ), and $\left\langle V_{e e}\right\rangle_{c}^{(2)}=$ $-0.10377 \mathrm{Ha}$ for the Hooke's series (to be compared with the exact ${ }^{16}$ value, $-0.09941 \mathrm{Ha})$. The error is thus $9 \mathrm{mH}$ for the He series and $4 \mathrm{mH}$ for the Hooke's series.

The correlation energy can then be otbained via the adiabatic connection formula of Eq. (1), which for $E_{c}^{(2)}$ reads

$$
E_{c}^{(2)}=\int_{0}^{\lambda_{\mathrm{phys}}} d \lambda \int_{0}^{\infty} d s_{12} 4 \pi s_{12}^{2} f_{c}^{\lambda(1)}\left(s_{12}\right) \frac{\partial w^{\lambda}\left(s_{12}\right)}{\partial \lambda}
$$

where $f_{c}^{\lambda(1)}$ is the first-order correlated part of $f$ for the system with interaction $\alpha w^{\lambda}\left(s_{12}\right)$. If we were able to calculate the exact $f_{c}^{\lambda(1)}$ for any $w^{\lambda}$, the resulting $E_{c}^{(2)}$ from Eq. (31) would be independent of the choice of $w^{\lambda}$. However, when we deal with approximations, we can obtain better results with some choices rather than others.

As in Ref. 7, we build an Overhauser-type potential along the adiabatic connection as

$$
w_{\mathrm{eff}}^{c, \lambda}\left(s_{12} ; \bar{s}_{s}\right)=w^{\lambda}\left(s_{12}\right)-\int_{|\mathbf{s}| \leq \bar{s}_{s}} \bar{n} w^{\lambda}\left(\left|\mathbf{s}-\mathbf{s}_{12}\right|\right) d \mathbf{s},
$$

where, in scaled units, if we stick with the choice of Eqs. (26)-(27), $\bar{n}=(4 \pi)^{-1}$ for the He series and $\bar{n}=\left(4 \pi^{3 / 2}\right)^{-1}$ for the Hooke's series. The idea behind 
Eq. (32) is that the average density $\bar{n}$ (and thus the average $\bar{s}_{s}$ ) is kept fixed to mimic the fact that the one-electron density does not change along the adiabatic connection while we turn on the e-e interaction.

\subsection{Linear adiabatic connection}

If we set $w^{\lambda}\left(s_{12}\right)=\lambda / s_{12}$, Eq. (32) simply gives the Overhauser potential of Eq. (28) with a multiplying factor $\lambda$ in front. From Eq. (23), we see that this corresponds to $E_{c}^{(2)}=\left\langle V_{e e}\right\rangle_{c}^{(2)} / 2$, as in the exact case. I.e., the simple approximation of Eq. (32) has the correct scaling behavior in the $\alpha \rightarrow 0$ limit. Our result for $E_{c}^{(2)}$ with the linear adiabatic connection thus gives an error of $4.5 \mathrm{mH}$ for the He series and $2 \mathrm{mH}$ for the Hooke's series.

\subsection{The "erf" adiabatic connection}

A choice for $w^{\lambda}$ that separates short- and long-range effects is the "erf" adiabatic connection, ${ }^{7,22-25} w^{\lambda}\left(s_{12}\right)=\operatorname{erf}\left(\lambda s_{12}\right) / s_{12}$, for which Eq. (31) becomes

$$
E_{c}^{(2)}=\int_{0}^{\infty} d \lambda \int_{0}^{\infty} d s_{12} 4 \pi s_{12}^{2} f_{c}^{\lambda(1)}\left(s_{12}\right) \frac{2}{\sqrt{\pi}} e^{-\lambda^{2} s_{12}^{2}} .
$$

The Overhauser-type potential corresponding to this interaction is reported in the appendix of Ref. 7. For the He isoelectronic series with $2 \leq Z \leq 10$, the Overhauser-type approximation combined with the "erf" adiabatic connection gives $^{7}$ correlation energies with errors within $4 \mathrm{mH}$, better than the linear adiabatic connection that gives errors within $10 \mathrm{mH}$.

In the weakly-correlated limit, instead, we obtained, via Eq. (33), $E_{c}^{(2)}=$ $-0.041 \mathrm{Ha}$ for the He series and $E_{c}^{(2)}=-0.046 \mathrm{Ha}$ for the Hooke's series. The errors with respect to the exact values, $6 \mathrm{mH}$ and $4 \mathrm{mH}$, respectively, are thus slightly worse than those obtained with the linear adiabatic connection.

\section{The LDA failure in the high-density limit: an analysis from the intracule density}

As a further element of comparison, we also computed the first-order $f_{c}^{(1)}\left(s_{12}\right)$ within the local-density approximation (LDA),

$$
f_{c}^{(1) \mathrm{LDA}}\left(s_{12}\right)=\lim _{\alpha \rightarrow 0} \frac{1}{\alpha} \int \frac{n^{(0)}(\mathbf{s})^{2}}{2} g_{c}\left(\tilde{k}_{F}(\mathbf{s}) s_{12} ; \alpha \tilde{r}_{s}(\mathbf{s})\right) d \mathbf{s},
$$

where $g_{c}\left(r_{12} ; r_{s}\right)$ is the pair-correlation function of the uniform electron gas ${ }^{26}$ of density $n=\left(4 \pi r_{s}^{3} / 3\right)^{-1}$, and

$$
\tilde{k}_{F}(\mathbf{s})=\left[3 \pi^{2} n^{(0)}(\mathbf{s})\right]^{1 / 3}, \quad \tilde{r}_{s}(\mathbf{s})=\left[\frac{4 \pi}{3} n^{(0)}(\mathbf{s})\right]^{-1 / 3} .
$$



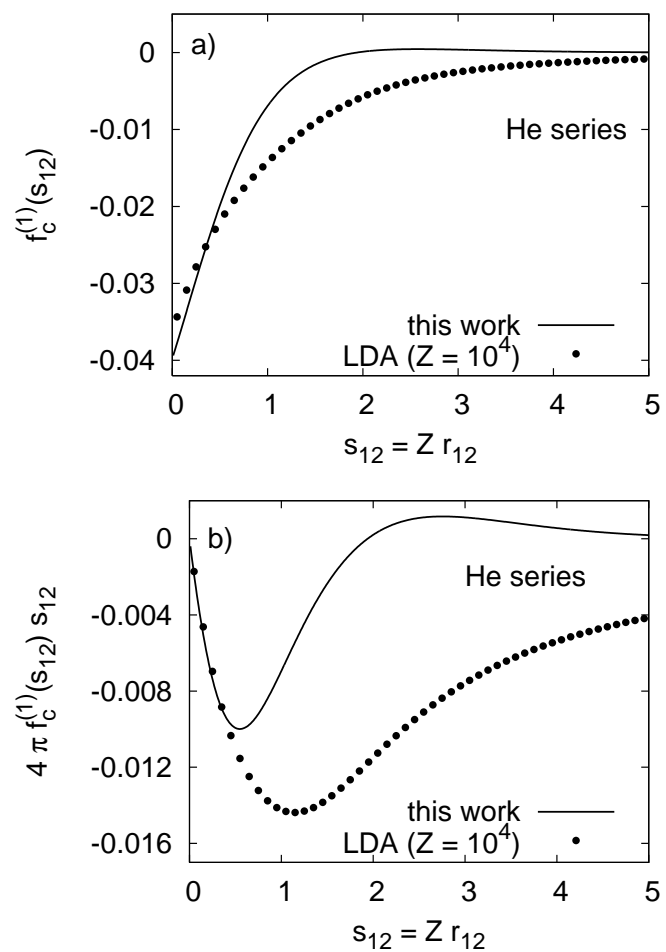

Figure 4: The correlated part of the intracule density, $f_{c}^{(1)}\left(s_{12}\right)$, of order $\alpha=1 / Z$ for the He series [see Eq. (12)]: the present calculation is compared with the LDA approximation (panel a). Panel b shows the same quantities multiplied by $4 \pi s_{12}$ : the integral under each curve gives the correlation part of the second order contribution to the expectation value $\left\langle V_{e e}\right\rangle$, which diverges in the case of LDA. 

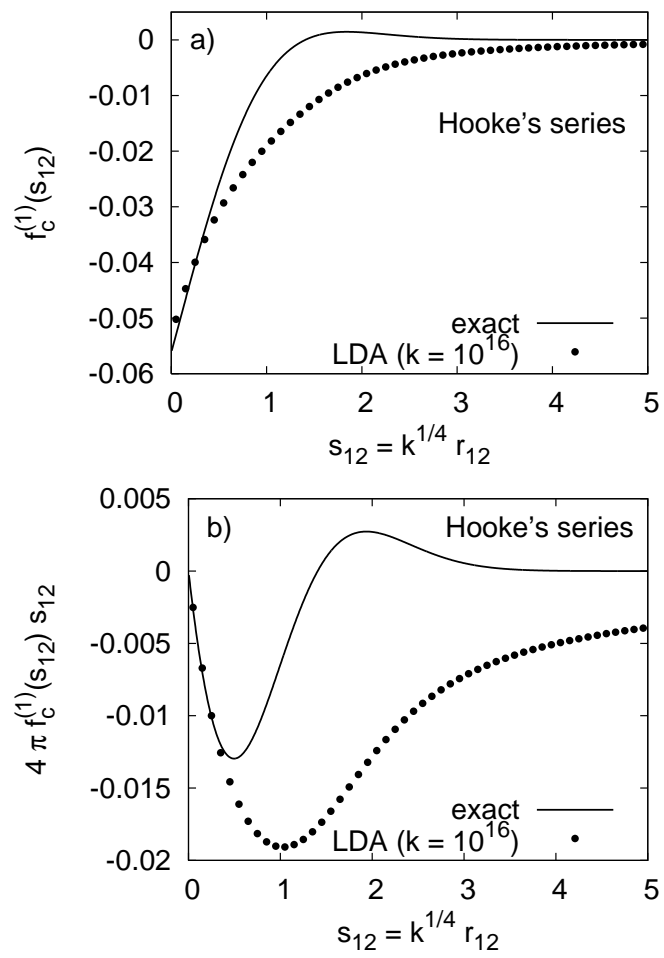

Figure 5: The correlated part of the intracule density, $f_{c}^{(1)}\left(s_{12}\right)$, of order $\alpha=$ $k^{-1 / 4}$ for the Hooke's atom series [see Eq. (12)]: the exact result is compared with the LDA approximation (panel a). Panel b shows the same quantities multiplied by $4 \pi s_{12}$ : the integral under each curve gives the correlation part of the second order contribution to the expectation value $\left\langle V_{e e}\right\rangle$, which diverges in the case of LDA. 
With these definitions, the density parameter $r_{s}$ of the uniform electron gas is locally proportional to $\alpha$. We have numerically evaluated the right-hand-side of Eq. (34) at smaller and smaller $\alpha$ (i.e., at larger and larger $Z$ and $k$ ), for $0 \leq s_{12} \leq 5$. As $\alpha$ decreases, the results tend to a well defined curve, shown in Figs. 4 and 5 , together with the result from the Overhauser model (He series) and the exact result (Hooke's series).

Since, as shown by Eq. (34), the $\alpha \rightarrow 0$ limit corresponds to the $r_{s} \rightarrow 0$ limit of the uniform electron gas pair-correlation function $g_{c}$, to better understand the LDA result for $f_{c}$ we now analyze more in detail the high-density behavior of $g_{c}$. This analysis extends and completes the one done in Ref. 27. When $r_{s} \rightarrow 0$, the short-range part of $g_{c}$ scales as

$$
g_{c}\left(x, r_{s} \rightarrow 0\right)=r_{s} g_{c}^{(1)}(x)+O\left(r_{s}^{2} \ln r_{s}\right), \quad x=r_{12} / r_{s},
$$

where the function $g_{c}^{(1)}(x)$ does not depend explictily on $r_{s}$ and has been computed by Rassolov et al. ${ }^{28}$ It is accurately recovered by the model $g_{c}$ of Ref. 26 that we have used in the evaluation of Eq. (34). The scaled variable $x$ is locally proportional to the scaled variable $s_{12}$ [see Eq. (35)]. Equation (36) thus shows that the short-range part (corresponding to values of the scaled variable $x$ not too large) of $g_{c}$ in the $r_{s} \rightarrow 0$ limit has a scaling similar to the one of the He and Hooke's series in the $\alpha \rightarrow 0$ limit. This is also reflected by a good performance of LDA for $s_{12} \lesssim 1$, as shown by Figs. 4 and 5 .

However, the high-density electron gas is an extended system with important long-range correlations that are not present in finite systems like atoms and molecules. In fact, the scaling of Eq. (36) is not valid when $x \gg 1$ : it has been shown that the long-range part of $g_{c}$ scales as ${ }^{26,29,30}$

$$
g_{c}\left(x \gg 1, r_{s}\right) \rightarrow r_{s}^{2} h(v),
$$

where $v$ is another scaled variable, $v=\sqrt{r_{s}} x$, which is thus locally proportional to $\sqrt{\alpha} s_{12}$. The function $h(v)$ has the following asymptotic behaviors:

$$
h(v \ll 1) \propto v^{-2}, \quad h(v \gg 1) \propto v^{-4},
$$

which are also correctly included in the model $g_{c}$ of Ref. 26. When $r_{s} \rightarrow 0$ (i.e., $\alpha \rightarrow 0$ ), even for very large $x$ the scaled variable $v$ is small, so that the long-range $(x \gg 1)$ behavior of $g_{c}$ is more and more dominated by the small $v$ part of $h(v)$, i.e., it behaves more and more like $v^{-2}$ rather than like $v^{-4}$. It is this increasing dominance of the "short-range component of the long-range part" that causes the $\propto \log \left(r_{s}\right)$ behavior in the correlation energy per electron of the high-density electron gas, and thus the divergence of the LDA correlation energy in the large- $Z$ and large- $k$ limit of the He and Hooke's atom sequences (see, e.g., Ref. 31). In fact, when $Z \rightarrow \infty$ (or $k \rightarrow \infty$ ), the high-density longrange behavior of $g_{c}$ affects the long-range part of $f_{c}^{\mathrm{LDA}}\left(s_{12}\right)$ in Eq. (34).

The small- $v$ behavior $\propto v^{-2}$ of the function $h(v)$ is related to the $1 / r_{12}$ divergence of the Coulomb potential at small $r_{12}$. For this reason, the $\propto \log \left(r_{s}\right)$ high-density behavior of the correlation energy is still present in a uniform 
electron gas with screened (or short-range only) Coulomb interaction (e.g., ${ }^{32}$ $\left.\operatorname{erfc}\left(\lambda r_{12}\right) / r_{12}\right)$, while is absent in an electron gas with long-range-only interaction (e.g., $\left.{ }^{33,34} \operatorname{erf}\left(\lambda r_{12}\right) / r_{12}\right)$.

\section{Conclusions}

We have computed the intracule density and the correlation energy for the high-density (weakly-correlated) limit of the He and Hooke's atom isoelectronic series via an approach ${ }^{7-9,17}$ based on an "average pair density functional theory" (APDFT), and inspired by the seminal work of Overhauser. ${ }^{10-13}$ Unlike the currently available correlation energy functionals analyzed in Ref. 14, the APDFT approach gives accurate results for both series. In its present formulation, the APDFT approach works well for two-electron systems and for the uniform electron gas. Its extension to many-electron systems of nonuniform density is a big challenge, and we are presently exploring several different paths to achieve this ambitious goal. ${ }^{9}$

We have also analyzed the LDA failure in the same weakly-correlated limit of the He and Hooke's atom series, in terms of the long-range part of the intracule density. The results of Katriel et al. ${ }^{14}$ show that higher-order functionals such as $\mathrm{PBE}^{35}$ and $\mathrm{TPSS}^{36}$ can reasonably fix the LDA problems in the case of the He isoelectronic series, but are much less satisfactory for the Hooke's atom sequence, yielding a wrong scaling in the $k \rightarrow \infty$ limit (PBE) or a correct scaling with an error of about $40 \%$ on the asymptotic value of the correlation energy (TPSS). As stressed by Katriel et al., ${ }^{14}$ these differences in performances for the two series raise serious doubts on the universality of currently available correlation energy functionals. The accuracy of the results obtained via the APDFT approach for both series suggests that the effort towards its generalization to many-electron systems of nonuniform density could be really worthwhile.

\section{Acknowledgments}

It is a pleasure to dedicate this methodological paper to Dennis Salahub, who did pioneering work not only in the applications of DFT, but also in the exploration of new methodologies in the DFT framework.

\section{A $\quad f_{\mathrm{KS}}^{(1)}\left(r_{12}\right)$ for the He isoelectronic series}

For the He isoelectronic series Eq. (13) corresponds to

$$
\begin{aligned}
f_{\mathrm{KS}}^{(1)}(x)= & \frac{1}{864 \pi x}\left\{4 e^{-4 x}[-41+3 x(1+9 x)]+81 e^{2 x}(x-1)[\operatorname{Ei}(-6 x)\right. \\
& -\operatorname{Ei}(-4 x)]+e^{-2 x}[164+27(3+x(9+4 x(3+2 x)))[\operatorname{Ei}(-2 x) \\
& -\gamma-\log (x)]+3 x[-163+6 x(15+x(7+10 x))-27 \log (4 / 3)] \\
& -162 \log (2)+81 \log (3)]\}
\end{aligned}
$$


where $\gamma$ and the function Ei have been defined after Eq. (91).

\section{References}

[1] Kohn, W. Rev. Mod. Phys. 1999, 71, 1253.

[2] Mattsson, A.E. Science 2002, 298, 759.

[3] Fiolhais, C.; Nogueira, F.; Marques, M. (eds.), A Primer in Density Functional Theory; Springer-Verlag: Berlin, 2003.

[4] Perdew, J. P.; Ruzsinszky, A.; Tao, J.; Staroverov, V. N.; Scuseria, G. E.; Csonka, G. I. J. Chem. Phys. 2005, 123, 062201.

[5] Harris, J.; Jones, R. J. Phys. F 1974, 4, 1170.

Langreth, D.C.; Perdew, J.P. Solid State Commun. 1975, 17, 1425.

Gunnarsson, O.; Lundqvist, B.I. Phys. Rev. B 1976, 13, 4274.

[6] Yang, W. J. Chem. Phys. 1998, 109, 10107.

[7] Gori-Giorgi P.; Savin, A. Phys. Rev. A. 2005, 71, 032513.

[8] Gori-Giorgi, P.; Savin, A. Philos. Mag. 2006, 86, 2643.

[9] Gori-Giorgi, P.; Savin, A. submitted to Int. J. Mod. Phys. B; arXiv:cond-mat/0611324 arXiv.org ePrint archive. http://arxiv.org/abs/cond-mat/0611324. (accessed Nov 13, 2006).

[10] Overhauser, A.W. Can. J. Phys. 1995, 73, 683.

[11] Gori-Giorgi, P.; Perdew, J.P. Phys. Rev. B 2001, 64, 155102.

[12] Davoudi, B.; Polini, M.; Asgari, R.; Tosi, M.P. Phys. Rev. B 2002, 66, 075110 .

[13] Corona, M.; Gori-Giorgi, P.; Perdew, J.P. Phys. Rev. B 2004, 69, 045108. Nagy, I.; Diez Muiño, R.; Juaristi, J.I.; Echenique, P.M. Phys. Rev. B 2004, 69, 233105 .

[14] Katriel, J.; Roy, S.; Springborg, M. J. Chem. Phys. 2006, 124, 234111.

[15] Schwartz, C. Ann. Phys. (N.Y.) 1959, 6, 156.

[16] White, R.J.; Byers Brown, W. J. Chem. Phys. 1970, 53, 3869.

Cioslowski, J.; Pernal, K. J. Chem. Phys. 2000, 113, 8434.

[17] Nagy, Á. J. Chem. Phys. 2006, 125, 184104.

[18] Dalgarno, A.; Lewis, J.T. Proc. Roy. Soc. (London) 1955, A233,70.

Dalgarno, A. Proc. Roy. Soc. (London) 1956, A69, 784.

Dalgarno A.; Stewart, A.L. Proc. Roy. Soc. (London) 1956 A238, 276. 
[19] Young, W.H.; March, N. H. Phys. Rev. 1958, 109, 1854.

[20] Freund, D.E.; Huxtable, B.D.; Morgan III, J.D. Phys. Rev. A 1984, 29, 980. We used an improved version (provided to us by C. Umrigar) of the accurate variational wavefunctions described in this work to obtain oneelectron densities $n(\mathbf{r})$ and functions $f\left(r_{12}\right)$. See also Umrigar, C.J.; Gonze, X. Phys. Rev. A 1994, 50, 3827.

[21] see, e.g., Huang, C.-J.; Umrigar, C.J. Phys. Rev. A 1997, 56, 290.

[22] Savin, A.; Colonna, F.; Pollet, R. Int. J. Quantum Chem. 2003, 93, 166, and references therein.

[23] Savin, A. in Recent Developments and Applications of Modern Density Functional Theory, edited by Seminario, J.M. Elsevier: Amsterdam, 1996; p. 327.

Leininger, T.; Stoll, H.; Werner, H.-J.; Savin, A. Chem. Phys. Lett. 1997, 275, 151.

Pollet, R. ; Savin, A.; Leininger, T.; Stoll, H. J. Chem. Phys. 2002, 116, 1250 .

[24] Pollet, R.; Colonna, F.; Leininger, T.; Stoll, H.; Werner, H.-J.; Savin, A. Int. J. Quantum Chem. 2003 91, 84.

[25] Toulouse, J.; Colonna, F.; Savin, A. Phys. Rev. A 2004, 70, 062505.

[26] Gori-Giorgi, P.; Perdew, J.P. Phys. Rev. B 2002, 66, 165118.

[27] Burke K.; Angulo J.C.; Perdew J.P. Phys. Rev. A 1994, 50, 297.

[28] Rassolov, V. A.; Pople, J. A.; Ratner, M. A. Phys. Rev. B 1999 59, 15625. Rassolov, V. A.; Pople, J. A.; Ratner, M. A. Phys. Rev. B 2000, 62, 2232.

[29] Wang, Y.; Perdew, J. P. Phys. Rev. B 1991, 44, 13298.

[30] Perdew, J.P.; Wang, Y. Phys. Rev. B 1992, 46, 12947.

[31] Perdew, J.P.; McMullen, E.R.; Zunger, A. Phys. Rev. A 1981 23, 2785.

[32] Zecca, L.; Gori-Giorgi, P.; Moroni, S.; Bachelet, G.B. Phys. Rev. B 2004, $70,205127$.

[33] Paziani, S.; Moroni, S.; Gori-Giorgi, P.; Bachelet, G.B. Phys. Rev. B 2006, 73, 155111.

[34] Toulouse, J. ; Gori-Giorgi, P.; Savin, A. Int. J. Quantum Chem. 2006, 106, 2026.

[35] Perdew J.P.; Burke K.; Ernzerhof M. Phys. Rev. Lett. 1996, 77, 3865.

[36] Tao J.; Perdew J.P.; Staroverov V.N.; Scuseria G.E. Phys. Rev. Lett. 2003, $91,146401$. 